PROCEEDINGS OF THE

AMERICAN MATHEMATICAL SOCIETY

Volume 125, Number 5, May 1997, Pages 1531-1536

S $0002-9939(97) 03826-4$

\title{
THE EXPECTED VALUE OF THE NUMBER OF REAL ZEROS OF A RANDOM SUM OF LEGENDRE POLYNOMIALS
}

\author{
J. ERNEST WILKINS, JR.
}

(Communicated by Richard T. Durrett)

\begin{abstract}
It is known that the expected number of zeros in the interval $(-1,1)$ of the sum $a_{0} \psi_{0}(t)+a_{1} \psi_{1}(t)+\cdots+a_{n} \psi_{n}(t)$, in which $\psi_{k}(t)$ is the normalized Legendre polynomial of degree $k$ and the coefficients $a_{k}$ are independent normally distributed random variables with mean 0 and variance 1 , is asymptotic to $3^{-1 / 2} n$ for large $n$. We improve this result and show that this expected number is $3^{-1 / 2} n+o\left(n^{\delta}\right)$ for any positive $\delta$.
\end{abstract}

\section{InTRODUCTION}

Let $k$ be a nonnegative integer, $P_{k}(t)$ be the Legendre polynomial of degree $k$, and $\psi_{k}(t)$ be the normalised Legendre polynomial $\left(k+\frac{1}{2}\right)^{\frac{1}{2}} P_{k}(t)$. Let $n$ be a positive integer, and consider the random sum

$$
F(t)=\sum_{k=0}^{n} a_{k} \psi_{k}(t),
$$

in which the coefficients $a_{k}$ are independent normally distributed random variables with mean 0 and variance 1 . If $\nu_{n}$ is the expected value of the number of zeros of $F(t)$ on the interval $(-1,1)$, then Das [1] has shown that $\nu_{n} \sim 3^{-\frac{1}{2}} n$ for large $n$. (In fact, his analysis indicates that $\nu_{n}=3^{-\frac{1}{2}} n\left[1+O\left\{(\log n)^{-3}\right\}\right]$.) In this paper we will prove the somewhat better result that

$$
\nu_{n}=3^{-\frac{1}{2}} n+o\left(n^{\delta}\right)
$$

for any positive $\delta$. Our analysis is similar to that of Das, but requires a more detailed treatment of the asymptotic expansion for $P_{n}(t)$ when $n$ is large.

\section{Preliminary analysis}

Let $\nu_{n}(a, b)$ be the expected number of zeros of $F(t)$ on the subinterval $(a, b)$ of $(-1,1)$. We know $([1]$ or $[2$, p. 111]) that

$$
\begin{gathered}
\nu_{n}(a, b)=\pi^{-1} \int_{a}^{b}\left\{A_{n}(t) C_{n}(t)-B_{n}^{2}(t)\right\}^{\frac{1}{2}} A_{n}^{-1}(t) d t \\
A_{n}(t)=P_{n+1}^{\prime}(t) P_{n}(t)-P_{n}^{\prime}(t) P_{n+1}(t)
\end{gathered}
$$

Received by the editors November 1, 1995.

1991 Mathematics Subject Classification. Primary 60G99; Secondary 41A60.

Key words and phrases. Real zeros, random polynomials, Legendre polynomials.

(C)1997 American Mathematical Society 


$$
2 B_{n}(t)=P_{n+1}^{\prime \prime}(t) P_{n}(t)-P_{n}^{\prime \prime}(t) P_{n+1}(t)
$$

$$
6 C_{n}(t)=3\left\{P_{n+1}^{\prime \prime}(t) P_{n}^{\prime}(t)-P_{n}^{\prime \prime}(t) P_{n+1}^{\prime}(t)\right\}+\left\{P_{n+1}^{\prime \prime \prime}(t) P_{n}(t)-P_{n}^{\prime \prime \prime}(t) P_{n+1}(t)\right\} .
$$

Stieltjes [3, 4] (or see [5, p. 195, Th. 8.21.5]) has shown that, if $0<\alpha<\pi$ and

$$
(n \sin \alpha)^{-1}=o(1)
$$

then

$(7)$

$$
P_{n}(\cos \alpha)=\left\{\frac{2}{(\pi \sin \alpha)}\right\}^{\frac{1}{2}}\left[\sum_{s=0}^{m-1} \frac{\gamma_{s} n ! \cos \beta_{n s}}{(2 \sin \alpha)^{s} \Gamma(n+s+3 / 2)}+O\left\{(n \sin \alpha)^{-m}\right\}\right],
$$

in which $\gamma_{s}=\left\{\Gamma\left(s+\frac{1}{2}\right)\right\}^{2} /(\pi s !), \beta_{n s}=\beta_{n s}(\alpha)=\left(n+s+\frac{1}{2}\right) \alpha-\frac{1}{2}\left(s+\frac{1}{2}\right) \pi$, and $m$ is any positive integer. Moreover, the upper bound implicit in the $O$ symbol depends only on $m$, not only in (7), but in the later identities (9), (10), (11), (14), (16), (18), (21), (22), (23) and (24). If we define $G_{k n}(\alpha)$ so that

$$
G_{k n}(\alpha)=\sum_{s=0}^{k} \gamma_{s} D_{k-s}(1, s+3 / 2)(2 \sin \alpha)^{k-s} \cos \beta_{n s},
$$

in which the coefficients $D_{h}(x, y)$ are those that appear in the asymptotic expansion [6, p. 119, Eq. 5.02]

$$
n^{y-x} \Gamma(n+x) / \Gamma(n+y)=\sum_{h=0}^{p-1} D_{h}(x, y) n^{-h}+O\left(n^{-p}\right),
$$

then $G_{k n}=G_{k n}(\alpha)$ is uniformly bounded in $n$ and $\alpha$, and we deduce from (7) that

$$
P_{n}(\cos \alpha)=\left\{\frac{2}{(n \pi \sin \alpha)^{\frac{1}{2}}}\right\}\left[\sum_{k=0}^{m-1}(2 n \sin \alpha)^{-k} G_{k n}+O\left(n^{-m} \sin ^{-m} \alpha\right)\right] .
$$

Because $D_{0}(x, y)=1$, we see that $G_{o n}(\alpha)=\cos \beta_{n o}$. Moreover,

$$
\begin{gathered}
P_{n+1}(\cos \alpha)=\left\{\frac{2}{(n \pi \sin \alpha)}\right\}^{\frac{1}{2}}\left[\sum_{k=0}^{m-1}(2 n \sin \alpha)^{-k} H_{k n}+O\left(n^{-m} \sin ^{-m} \alpha\right)\right] \\
P_{n-1}(\cos \alpha)=\left\{\frac{2}{(n \pi \sin \alpha)}\right\}^{\frac{1}{2}}\left[\sum_{k=0}^{m-1}(2 n \sin \alpha)^{-k} J_{k n}+O\left(n^{-m} \sin ^{-m} \alpha\right)\right] \\
H_{k n}=\sum_{s=0}^{k}-s-\frac{1}{2} C_{k-s}(2 \sin \alpha)^{k-s} G_{s, n+1}, \\
J_{k n}=\sum_{s=0}^{k}-s-\frac{1}{2} C_{k-s}(-2 \sin \alpha)^{k-s} G_{s, n-1},
\end{gathered}
$$

in which ${ }_{q} C_{j}=\Gamma(j+1) /\{\Gamma(q+1) \Gamma(j-q+1)\}$. The functions $H_{k n}$ and $J_{k n}$ are uniformly bounded in $n$ and $\alpha$, and $H_{o n}=\cos \beta_{n+1, o}, J_{o n}=\cos \beta_{n-1, o}$.

We use the identity $[7$, p. 309, eq. V]

$$
\left(1-t^{2}\right) P_{n}^{\prime}(t)=n\left\{P_{n-1}(t)-t P_{n}(t)\right\}
$$


in conjunction with (9) and (11) to see that

$$
\begin{gathered}
P_{n}^{\prime}(\cos \alpha)=\left\{\frac{2 n}{\left(\pi \sin ^{3} \alpha\right)}\right\}^{\frac{1}{2}}\left[\sum_{k=0}^{m-1}(2 n \sin \alpha)^{-k} K_{k n}+O\left(n^{-m} \sin ^{-m-1} \alpha\right)\right] \\
K_{k n}=\left(J_{k n}-G_{k n} \cos \alpha\right) / \sin \alpha .
\end{gathered}
$$

It is now convenient to define a congruence relation $\equiv$ between two functions $X$ and $Y$ of $n$ and $\alpha$ so that $X \equiv Y$ in case $(X-Y) / \sin \alpha$ is uniformly bounded in $n$ and $\alpha$. This relation is an equivalence relation that is preserved under addition and under multiplication by uniformly bounded functions. It follows from (13) that $J_{k n} \equiv G_{k, n-1}$ and from (8) that $G_{k n} \equiv \gamma_{k} \cos \beta_{n k}$. Hence $J_{n k}-G_{n k} \cos \alpha \equiv$ $\gamma_{k}\left(\cos \beta_{n-1, k}-\cos \beta_{n k} \cos \alpha\right)=\gamma_{k} \sin \beta_{n k} \sin \alpha$, so that the function $K_{k n}$ defined in (15) is uniformly bounded in $n$ and $\alpha$. In particular, $K_{o n}=\sin \beta_{n o}$. Moreover,

$$
\begin{gathered}
P_{n+1}^{\prime}(\cos \alpha)=\left\{\frac{2 n}{\left(\pi \sin ^{3} \alpha\right)}\right\}^{\frac{1}{2}}\left[\sum_{k=0}^{m-1}(2 n \sin \alpha)^{-k} L_{k n}+O\left(n^{-m} \sin ^{-m-1} \alpha\right)\right] \\
L_{k n}=\sum_{s=0}^{k} \frac{1}{2}-s C_{k-s}(2 \sin \alpha)^{k-s} K_{s, n+1} .
\end{gathered}
$$

Therefore, $L_{k n}$ is uniformly bounded in $n$ and $\alpha$, and $L_{o n}=\sin \beta_{n+1, o}$.

It now follows from (3), (9), (10), (14) and (16) that, if $t=\cos \alpha$,

$$
\begin{gathered}
A_{n}(t)=\left\{\frac{2 n}{(\pi \sin \alpha)}\right\}\left[\sum_{k=0}^{m-1}(2 n \sin \alpha)^{-k} M_{k n}+O\left(n^{-m} \sin ^{-m-2} \alpha\right)\right], \\
M_{k n}=\sum_{s=0}^{k}\left(L_{s n} G_{k-s, n}-K_{s n} H_{k-s, n}\right) / \sin \alpha .
\end{gathered}
$$

We deduce from (17), (8) and (12) that

$$
\begin{aligned}
L_{s n} G_{k-s, n}-K_{s n} H_{k-s, n} & \equiv \gamma_{k-s}\left(K_{s, n+1} \cos \beta_{n, k-s}-K_{s n} \cos \beta_{n+1, k-s}\right) \\
& \equiv \gamma_{k-s}\left(K_{s, n+1}-K_{s n} \cos \alpha\right) \cos \beta_{n, k-s} .
\end{aligned}
$$

We infer from (15), (13) and (8) that $K_{s n}=U_{s n}^{\prime}+U_{s n}^{\prime \prime}$, in which

$$
\begin{aligned}
U_{s n}^{\prime} & =\left(J_{s n}-G_{s, n-1}\right) / \sin \alpha=\sum_{k=0}^{s-1}-k-\frac{1}{2} C_{s-k}(-2 \sin \alpha)^{s-1-k} G_{k, n-1} \\
& \equiv-\left(s-\frac{1}{2}\right) G_{s-1, n-1} \equiv-\left(s-\frac{1}{2}\right) \cos \beta_{n-1, s-1}, \\
U_{s n}^{\prime \prime} & =\left(G_{s, n-1}-G_{s n} \cos \alpha\right) / \sin \alpha \\
& =\sum_{k=0}^{s} \gamma_{r} D_{s-k}(1, k+3 / 2)(2 \sin \alpha)^{s-k} \sin \beta_{n k} \equiv \gamma_{s} \sin \beta_{n s} .
\end{aligned}
$$

We next observe that

$$
\begin{aligned}
U_{s, n+1}^{\prime}-U_{s n}^{\prime} \cos \alpha & \equiv-\left(s-\frac{1}{2}\right)\left(\cos \beta_{n, s-1}-\cos \beta_{n-1, s-1} \cos \alpha\right) \\
& =\left(s-\frac{1}{2}\right) \sin \beta_{n-1, s-1} \sin \alpha \equiv 0 \\
U_{s, n+1}^{\prime \prime}-U_{s n}^{\prime \prime} \cos \alpha & \equiv \gamma_{s}\left(\sin \beta_{n+1, s}-\sin \beta_{n s} \cos \alpha\right) \\
& =\gamma_{s} \cos \beta_{n s} \sin \alpha \equiv 0
\end{aligned}
$$


It follows from (19) that $M_{k n}$ is uniformly bounded in $n$ and $\alpha$, and $M_{o n}=1$.

It is a consequence of the differential equation [7, p. 304]

$$
\left(1-t^{2}\right) P_{n}^{\prime \prime}(t)-2 t P_{n}^{\prime}(t)+n(n+1) P_{n}(t)=0,
$$

satisfied by $P_{n}(t)$, and the definitions (3) and (4) that

$$
\left(1-t^{2}\right) B_{n}(t)=t A_{n}(t)-(n+1) P_{n}(t) P_{n+1}(t) .
$$

It then follows from (18), (9) and (10) that

$$
B_{n}(t)=\left\{\frac{2}{\left(\pi \sin ^{3} \alpha\right)}\right\}\left[\sum_{k=0}^{m-1}(2 n \sin \alpha)^{-k} N_{k n}+O\left(n^{-m} \sin ^{-m-2} \alpha\right)\right],
$$

in which $N_{k n}$ is a function, uniformly bounded in $n$ and $\alpha$, whose explicit expression is not needed.

An additional consequence of (20) is that (it is convenient to suppress the dependence on $t$ of the Legendre polynomials and their derivatives)

$$
\left(1-t^{2}\right)\left(P_{n+1}^{\prime \prime} P_{n}^{\prime}-P_{n}^{\prime \prime} P_{n+1}^{\prime}\right)=(n+1)\left(n A_{n}-2 P_{n+1} P_{n}^{\prime}\right) .
$$

If we differentiate (20) and use the definitions (3) and (4), we find that

$$
\left(1-t^{2}\right)\left(P_{n+1}^{\prime \prime \prime} P_{n}-P_{n}^{\prime \prime \prime} P_{n+1}\right)=n^{2} A_{n}+8 t B_{n}+(n-2) P_{n+1} P_{n}^{\prime}-3 n P_{n+1}^{\prime} P_{n} .
$$

It then follows from (5) and the last two equations that

$$
6\left(1-t^{2}\right) C_{n}=\left(2 n^{2}+3 n\right) A_{n}+8 t B_{n}-(5 n+8) P_{n+1} P_{n}^{\prime}-3 n P_{n+1}^{\prime} P_{n} .
$$

An appeal to (18), (21), (10), (14), (16) and (7) shows that

$$
C_{n}(t)=\left\{\frac{2 n^{2}}{\left(3 \pi \sin ^{3} \alpha\right)}\right\}\left[\sum_{k=0}^{m-1}(2 n \sin \alpha)^{-k} Q_{k n}+O\left(n^{-m} \sin ^{-m-2} \alpha\right)\right],
$$

in which $Q_{k n}$ is a function, uniformly bounded in $n$ and $\alpha$, whose explicit expression is not needed, except for the case $Q_{o n}=1$.

With the help of (18), (21) and (22), we now find that

$$
\begin{aligned}
& \left\{A_{n}(t) C_{n}(t)-B_{n}^{2}(t)\right\}^{\frac{1}{2}} A_{n}^{-1}(t) \\
& \quad=\left(\frac{3^{-\frac{1}{2}} n}{\sin \alpha}\right)\left[\sum_{k=0}^{m-1}(2 n \sin \alpha)^{-k} R_{k n}+O\left(n^{-m} \sin ^{-m-2} \alpha\right)\right],
\end{aligned}
$$

in which $R_{k n}$ is a function, uniformly bounded in $n$ and $\alpha$, whose explicit expression is not needed, except for the case $R_{o n}=1$.

\section{Proof of (1)}

Suppose that $\varepsilon=n^{-2 m /(m+4)}$, and that $|t| \leq 1-\varepsilon$. Then $n \sin \alpha=n\left(1-t^{2}\right)^{\frac{1}{2}}>$ $n \varepsilon^{\frac{1}{2}}=n^{4 /(m+4)}$. Hence (6) is true. We conclude from (2) and (23) that

$$
\begin{aligned}
\nu_{n}(-1 & +\varepsilon, 1-\varepsilon)=2 \nu_{n}(0,1-\varepsilon) \\
=\left\{\frac{2 n}{\left(3^{\frac{1}{2}} \pi\right)}\right\} \int_{0}^{1-\varepsilon}\left[\left(1-t^{2}\right)^{-\frac{1}{2}}\right. & +\sum_{k=1}^{m-1} O\left\{n^{-k}\left(1-t^{2}\right)^{-(k+1) / 2}\right\} \\
& \left.+O\left\{n^{-m}\left(1-t^{2}\right)^{-(m+5) / 2}\right\}\right] d t .
\end{aligned}
$$


We observe that

$$
\begin{gathered}
\int_{0}^{1-\varepsilon}\left(1-t^{2}\right)^{-\frac{1}{2}} d t=(\pi / 2)-\cos ^{-1}(1-\varepsilon)=(\pi / 2)+O\left(\varepsilon^{\frac{1}{2}}\right), \\
\int_{0}^{1-\varepsilon}\left(1-t^{2}\right)^{-1} d t=\frac{1}{2} \log \{(2-\varepsilon) / \varepsilon\}=O\left(\log \varepsilon^{-1}\right) \\
\int_{0}^{1-\varepsilon}\left(1-t^{2}\right)^{-3 / 2} d t=(1-\varepsilon)\left(2 \varepsilon-\varepsilon^{2}\right)^{-\frac{1}{2}}=O\left(\varepsilon^{-\frac{1}{2}}\right) .
\end{gathered}
$$

Because $1-t^{2}>\varepsilon$ when $0 \leq t \leq 1-\varepsilon$, we see that

$$
\int_{0}^{1-\varepsilon}\left(1-t^{2}\right)^{\left(-h-\frac{1}{2}\right)} d t<\varepsilon^{(1-h)} \int_{0}^{1-\varepsilon}\left(1-t^{2}\right)^{-3 / 2} d t=O\left(\varepsilon^{-h+\frac{1}{2}}\right)
$$

when $h \geq 1$. Therefore,

$$
\begin{aligned}
\nu_{n}(-1+\varepsilon, 1-\varepsilon)=3^{-\frac{1}{2}} n[1+ & O\left(\varepsilon^{\frac{1}{2}}\right)+O\left(n^{-1} \log \varepsilon^{-1}\right) \\
& \left.+\varepsilon^{\frac{1}{2}} \sum_{k=2}^{m-1} O\left\{\left(n \varepsilon^{\frac{1}{2}}\right)^{-k}\right\}+O\left(n^{-m} \varepsilon^{-(m+3) / 2}\right)\right], \\
\nu_{n}(-1+\varepsilon, 1-\varepsilon) & =3^{-\frac{1}{2}} n\left[1+O\left\{n^{-m /(m+4)}\right\}\right] .
\end{aligned}
$$

Let $\mu(\varepsilon)$ be the number of complex zeros of $F(t)$ in the circle $|t-1|<\varepsilon$. Then $\nu_{n}(1-\varepsilon, 1)$ does not exceed the expected value of $\mu(\varepsilon)$. It follows from Jensen's Theorem [8, p. 187, Eq. 25]

$$
\mu(\varepsilon) \leq(2 \pi)^{-1} \int_{0}^{2 \pi} \log _{2}\left|F\left(1+2 \varepsilon e^{i \theta}\right) / F(1)\right| d \theta .
$$

We next use the identity [7, p. 312],

$$
P_{k}(z)=\pi^{-1} \int_{0}^{\pi}\left\{z+\left(z^{2}-1\right)^{\frac{1}{2}} \cos \varphi\right\}^{k} d \varphi
$$

to see that

$$
\left|P_{k}\left(1+2 \varepsilon e^{i \theta}\right)\right| \leq\left\{1+2 \varepsilon+2\left(\varepsilon+\varepsilon^{2}\right)^{\frac{1}{2}}\right\}^{k}<\left(1+A \varepsilon^{\frac{1}{2}}\right)^{k},
$$

in which $A=2\left(1+2^{\frac{1}{2}}\right)<5$. Therefore, when $0 \leq k \leq n$,

$$
\left|P_{k}\left(1+2 \varepsilon e^{i \theta}\right)\right|<\exp \left\{n \log \left(1+5 \varepsilon^{\frac{1}{2}}\right)\right\}<\exp \left(5 n \varepsilon^{\frac{1}{2}}\right) .
$$

The Chebyshev inequality $[9$, p. 219, Eq. 61$]$ shows that $\operatorname{Prob}\left(\left|a_{k}\right| \leq n\right)>1-n^{-2}$ for each $k$, such that

$$
\operatorname{Prob}\left(\left|a_{k}\right| \leq n \text { when } 0 \leq k \leq n\right)>1-(n+1) n^{-2} \geq 1-2 n^{-1} .
$$

Because the Schwarz inequality implies that

$$
\sum_{k=0}^{n}\left(k+\frac{1}{2}\right)^{\frac{1}{2}} \leq\left\{(n+1) \sum_{k=0}^{n}\left(k+\frac{1}{2}\right)\right\}^{\frac{1}{2}}=\left\{(n+1)^{3} / 2\right\}^{\frac{1}{2}}<n^{3 / 2}
$$

when $n \geq 4$, it follows from (26) and (27) that

$$
\operatorname{Prob}\left\{\left|F\left(1+2 \varepsilon e^{i \theta}\right)\right|<n^{5 / 2} \exp \left(5 n \varepsilon^{\frac{1}{2}}\right)\right\}>1-2 n^{-1} .
$$


Moreover, $F(1)$ is a normally distributed random variable with mean 0 and variance $\zeta^{2}=(n+1)^{2} / 2$. Therefore,

$$
\operatorname{Prob}\{|F(1)|<1\}=\left(2 \pi \zeta^{2}\right)^{-\frac{1}{2}} \int_{-1}^{1} \exp \left(-u^{2} / 2 \zeta^{2}\right) d u<2 \pi^{-\frac{1}{2}} /(n+1)<2 n^{-1} .
$$

We infer from $(25),(28)$ and $(29)$ that $\mu(\varepsilon)<\log _{2}\left\{n^{5 / 2} \exp \left(5 n \varepsilon^{\frac{1}{2}}\right)\right\}$ with probability greater than $1-4 n^{-1}$. Because $\mu(\varepsilon) \leq n$ for all $F(t)$, we see that the expected value of $\mu(\varepsilon)$ is, and so also $\nu_{n}(1-\varepsilon, 1)$ and $\nu_{n}(-1,-1+\varepsilon)$ are, $O(\log n)+O\left(n \varepsilon^{\frac{1}{2}}\right)+O(1)=$ $O\left(n^{4 /(m+4)}\right)$. When this result is combined with $(24)$, we find that $\nu_{n}=\nu_{n}(-1,1)=$ $3^{-\frac{1}{2}} n+O\left\{n^{4 /(m+4)}\right\}$. If $\delta$ is any positive number and we choose the integer $m$ so large that $m+4>4 / \delta$, we can finally conclude that (1) is true, in the sense that for any positive $\eta$ and $\delta$ there exists an integer $n(\eta, \delta)$ such that $\left|\nu_{n}-3^{-\frac{1}{2}} n\right| n^{-\delta}<\eta$ when $n>n(\eta, \delta)$.

\section{REFERENCES}

1. M. Das, Real zeros of a random sum of orthogonal polynomials, Proc. Amer. Math. Soc. 27 (1971), 147-153. MR 42:3830

2. A. T. Bharucha-Reid and M. Sambandham, Random Polynomials, Academic Press, Orlando, 1986. MR 87 m:60118

3. T. J. Stieltjes, Sur la valeur asymptotique des polynomes de Legendre, Comptes Rendu de l'Academie des Sciences, Paris 110 (1890), 1026-1027.

4. T. J. Stieltjes, Sur les polynomes de Legendre, Annales de la Faculté des Sciences de Toulouse, 4 (1890), 17 pages.

5. G. Szego, Orthogonal Polynomials, Amer. Math. Soc. Colloquium Publications, XXIII, Providence, Rhode Island, 1939. MR 1:14b

6. F. W. J. Olver, Asymptotics and Special Functions, Academic Press, New York, 1974. MR 55:8655

7. E. T. Whittaker and G. N. Watson, A Course of Modern Analysis, American Edition, Cambridge University Press, Cambridge, 1943. MR 31:2375

8. L. V. Ahlfors, Complex Analysis, McGraw Hill Book Co., New York, 1953. MR 14:857a

9. W. Feller, An Introduction, n to Probability Theory and its Applications, Vol. I, 2nd Edition, John Wiley and Sons, New York, 1957. MR 19:466a

Department of Mathematics, Clark Atlanta University, Atlanta, Georgia 30314 